\title{
Cellulose nanofibril film as a piezoelectric sensor
}

\section{material}

Satu Rajala ${ }^{1 *}$, Tuomo Siponkoski ${ }^{2}$, Essi Sarlin ${ }^{3}$, Marja Mettänen ${ }^{1}$, Maija Vuoriluoto $^{4}$, Arno

Pammo $^{1}$, Jari Juuti ${ }^{2}$, Orlando J. Rojas ${ }^{4}$, Sami Franssila ${ }^{5}$ and Sampo Tuukkanen ${ }^{1 \dot{f}^{*}}$

${ }^{1}$ Department of Automation Science and Engineering, Tampere University of Technology, P.O.

Box 692, FI-33101 Tampere, Finland, satu.rajala@tut.fi, marja.mettanen@tut.fi, arno.pammo@tut.fi,sampo.tuukkanen@tut.fi

${ }^{2}$ Microelectronics Research Unit, Faculty of Information Technology and Electrical Engineering, University of Oulu, P.O. Box 4500, FIN-90014 Oulu, Finland, tuomo.siponkoski@oulu.fi,jari.juuti@oulu.fi

${ }^{3}$ Department of Materials Science, Tampere University of Technology, P.O. Box 692, FI-33101 Tampere, Finland, essi.sarlin@tut.fi

${ }^{4}$ Department of Forest Products Technology, School of Chemical Technology, Aalto University, P.O. Box 16300, FI-00076 Aalto, Finland, maija.vuoriluoto@aalto.fi, orlando.rojas@aalto.fi

${ }^{5}$ Department of Materials Science and Engineering, School of Chemical Technology, Aalto University, P.O. Box 11000, FI-00076 Aalto, Espoo, Finland, sami.franssila@aalto.fi 
Keywords: Cellulose nanofibrils; nanocellulose films; piezoelectric sensor materials; imagebased analysis; relative permittivity; sensitivity measurement.

\begin{abstract}
Self-standing films (45- $\mu \mathrm{m}$ thick) of native cellulose nanofibrils (CNF) were synthesized and characterized for their piezoelectric response. The surface and the microstructure of the films were evaluated with image-based analysis and scanning electron microscopy (SEM). The measured dielectric properties of the films at $1 \mathrm{kHz}$ and $9.97 \mathrm{GHz}$ indicated a relative permittivity of 3.47 and 3.38 and loss tangent $\tan \delta$ of 0.011 and 0.071 , respectively. The films were used as functional sensing layers in piezoelectric sensors with corresponding sensitivities of 4.7 to $6.4 \mathrm{pC} / \mathrm{N}$ in ambient conditions. This piezoelectric response is expected to increase remarkably upon film polarization resulting from the alignment of the cellulose crystalline regions in the film. The CNF sensor characteristics were compared with those of polyvinylidene fluoride (PVDF) as reference piezoelectric polymer. Overall, the results suggest that CNF is a suitable precursor material for disposable piezoelectric sensors, actuator or energy generators with potential applications in the fields of electronics, sensors and biomedical diagnostics.
\end{abstract}

\title{
1. Introduction
}

Cellulosic nanofibrils and nanocrystals ${ }^{1}$, referred to as nanocelluloses, are interesting renewable bio-based nanomaterials with potential applications in different fields. The nanoscale dimensions and strong ability to form entangled porous networks make nanocelluloses suitable 
materials for fabrication of light-weight membranes, films, and nanopapers, all of which can be processed in aqueous media. Processing in aqueous media ensures low cost and high throughput manufacturing of functional devices for electronics ${ }^{2,3}$, sensing ${ }^{4}$ and optics ${ }^{5}$. Furthermore, the combination of nanocelluloses with water-processable carbon-based nanomaterials, such as carbon nanotubes or graphene, is beneficial towards flexible and disposable devices, including supercapacitors $^{6-8}$ among many others.

The piezoelectricity of wood, i.e., the change of electrical polarization in a material in response to mechanical stress ${ }^{9}$, has been known for decades ${ }^{10,11}$. The piezoelectric effect is highly enhanced if one considers the isolated crystalline building blocks of wood, namely cellulose nanocrystals $(\mathrm{CNC})^{12,13}$. However, related issues has been covered in the scientific literature to a very limited extent and only few recent reports discuss experimental evidence of CNC piezoelectricity $^{12,14}$. Notably, the higher aspect-ratio cellulose nanofibrils (CNF), containing both crystalline (cellulose $\mathrm{I}^{1}$ ) and less ordered domains of cellulose, may offer versatile options as far as the synthesis of self-standing films and nanopapers is profitable. Moreover, despite the expected random alignment of crystalline regions in $\mathrm{CNF}$, their films may still be piezoelectric due to the film fabrication process driven alignment. To our knowledge, piezoelectric measurements of CNF films have not been attempted before us ${ }^{15,16}$.

In this study, CNF films were fabricated and their physical, dielectric and electromechanical behavior was characterized. The microstructure of the films was studied using scanning electron microscopy (SEM). Also, the surface of the films was evaluated through measurements based on 
optical imaging. The films were used as functional materials in piezoelectric sensors and their sensitivity was measured by using a custom-built setup. The sensors were excited with a mechanical shaker that delivered a sinusoidal force input and the charge generated by the sensor was measured. The sensitivity is defined here as the charge generated by the sensor divided by the force applied to excite the sensor. Finally, the nonlinearity and hysteresis of the piezoelectric sensors were measured and compared with those of polyvinylidene fluoride (PVDF) which was used as a reference piezoelectric polymer.

\section{Materials and methods}

\subsection{Nanocellulose Piezoelectricity}

The compression of a piezoelectric film by an external force causes charge separation in the film generating a noticeable voltage between two electrodes. The piezoelectric coefficient $d_{m n}$ is related to the charge density generated under a certain applied stress. The components of thirdrank tensor with piezoelectric coefficients $d_{m n}$ is expressed as a $3 \times 6$ matrix, where $m=1,2,3$ refers to the electrical axis and $n=1,2, \ldots, 6$ to the mechanical axis ${ }^{9}$. The main axes 1,2 and 3 correspond to length, width and thickness, whereas the shear around these axes is represented by 4,5 and 6.

The piezoelectric tensor $d_{m n}$ can be derived from the symmetry of a cellulose crystal lattice, formed by unit cells of cellulose molecules $\left(\left[\mathrm{C}_{6} \mathrm{H}_{10} \mathrm{O}_{5}\right]_{n}\right)^{11}$. The cellulose belongs to the monoclinic symmetry with space group of $\mathrm{C}_{2} \| \mathrm{x}_{3}$ having a following piezoelectric tensor: 


$$
d_{m n}=\left(\begin{array}{cccccc}
0 & 0 & 0 & d_{14} & d_{15} & 0 \\
0 & 0 & 0 & d_{24} & d_{25} & 0 \\
d_{31} & d_{32} & d_{33} & 0 & 0 & d_{36}
\end{array}\right) .
$$

This tensor is valid for a single cellulose crystal. However, for an assembly of randomly aligned crystals, such as in a film of $\mathrm{CNF}$, the overall piezoelectric response results from the combination of the different coefficients. For example, in the case of wood, arrangement of fibers and accompanied cellulose crystals have been shown to exhibit greatly reduced effective piezoelectric tensor:

$$
d_{m n}=\left(\begin{array}{cccccc}
0 & 0 & 0 & d_{14} & 0 & 0 \\
0 & 0 & 0 & 0 & d_{25} & 0 \\
0 & 0 & 0 & 0 & 0 & 0
\end{array}\right)
$$

where $d_{14}=-d_{25}{ }^{10,11}$.

\subsection{CNF film fabrication}

Figure 1 describes schematically the deconstruction of nanocellulose from wood cellulose fibers $^{1}$. In this work, fibers obtained from birch wood by sulfate digestion and subsequent bleaching were suspended in water and then processed through a Masuko grinder using three consecutive passes and further homogenized using six passes at 2000 bar pressure by using a M110P microfluidizer (Microfluidics Corp., Newton, MA, USA) equipped with two chambers (200 and $100 \mu \mathrm{m}$ size) in series. The resulting cellulose fibrils can be considered as composed of ordered crystalline (cellulose $\mathrm{I}^{1}$ ) and less ordered cellulose domains as well as amorphous residual heteropolysaccharides and lignin, which may be present in very low concentration. Even 
at low $\mathrm{CNF}$ concentration, it forms a highly viscous aqueous dispersion, a hydroge ${ }^{17}$. Films based on CNF were fabricated by pressure filtering (15-30 min) followed by pressing and drying in a hot-press at $100{ }^{\circ} \mathrm{C}$ for $2 \mathrm{~h}$, as has been described previously in detail ${ }^{18}$. An example of the resulting self-standing CNF films is presented in Figure 2a.

\subsection{CNF film characterization}

The CNF films were characterized by Scanning Electron Microscopy (SEM, Zeiss ULTRAplus). Cross-sectional images were obtained by cryo-fracture of the CNF film under liquid nitrogen and by gluing the film to holders containing conductive carbon cement. The samples were coated with a thin carbon layer to provide electrical conductivity prior to SEM imaging. Energy Dispersive X-ray Spectroscopy (EDS, INCA Energy 350 with INCAx-act detector) was used to analyse the purity of the CNF film. The apparent film density was calculated from a $5 \mathrm{~cm} \times 8 \mathrm{~cm}$ cut by assuming an even film thickness (as obtained from the SEM analysis) and mass (microbalance).

The surface topography of the CNF film was examined through photometric stereo imaging and subsequent image analysis. Photometric stereo ${ }^{19}$ imaging is a widely used technique in computer graphics for surface analysis and reconstruction ${ }^{20}$, and it has also been successfully applied in small-scale surface topography measurement, e.g., in paper products ${ }^{21}$ and dermatology $\mathrm{y}^{22}$. In the photometric stereo setup, the translucent CNF sample was fixed on top of a flat black sheet of cardboard while it was imaged with a camera placed atop. Four images were acquired from the sample with different, but precisely known, illumination directions, using blue 
LEDs whose dominant wavelength was $460 \mathrm{~nm}$. The surface orientation (i.e., gradients) at each pixel was estimated from the photographs (see Ref. ${ }^{19}$ for details). The surface topography map was then reconstructed from the gradient fields by integration ${ }^{23}$. The photographs were captured using a digital systems camera (Canon EOS 550D) and a magnifying macro lens (Canon MP-E $65 \mathrm{~mm}$ ). With a slight magnification (1.3:1), a pixel size of $3.27 \mu \mathrm{m} \times 3.27 \mu \mathrm{m}$ on an image area of approximately $17 \mathrm{~mm} \times 11 \mathrm{~mm}$ was achieved.

The dielectric properties of the CNF film were evaluated at low frequency (100 Hz to $1 \mathrm{MHz}$ ) by using a LCR meter (precision LCR meter 4284A, Keysight Technologies, USA). Frequencies below $100 \mathrm{~Hz}$ were left out from the measurement due to low accuracy of the LCR at these frequencies and capacitance values below $100 \mathrm{pF}$. In order to improve the reliability of the measurement, a stack of two films ( $45 \mu \mathrm{m}$ each for a total thickness of $96 \mu \mathrm{m}$ ) was used. The sample was compressed gently between two external electrodes (diameters 25 and $17.5 \mathrm{~mm}$ ) in a custom-made measurement setup. The holder of the smaller electrode was made from soft rubber, covered with a conductive layer. This enabled the sample to align better between the electrodes and improved the application of small pressures to avoid deformation of the sample. Further, a high frequency analysis was used to confirm the reliability of the LCR measurements; thus, a single layer of the CNF film was characterized at $9.97 \mathrm{GHz}$ using a Split Post Dielectric Resonator (QWED, Warsaw, Poland). This technique enables measurement without electrodes. Prior to both dielectric measurements the films were held at $100{ }^{\circ} \mathrm{C}$ and equilibrated for $60 \mathrm{~min}$ in order to minimize the effect of air moisture in the results. Ferroelectric tester (Precision LC, Radiant technologies, USA) was used to measure the ferroelectric hysteresis of the CNF film up to $50 \mathrm{~V} / \mu \mathrm{m}$ electric field and $10 \mathrm{~Hz}$ frequency. 


\subsection{CNF and PVDF sensor assembly}

Electrode substrates for the CNF sensor assembly were fabricated with polyethylene terephthalate (PET, Melinex ST506) by e-beam evaporation (Varian vacuum evaporator). Electrodes were formed by evaporating $100 \mathrm{~nm}$ thick copper $(\mathrm{Cu})$ layer through a laser-cut stencil shadow mask.

The preparation of piezoelectric CNF sensor is described in Figure 2. Before the sensor assembly, the CNF film shown in Figure 2a was cut into round shapes with a diameter slightly larger than that of the electrodes, thus to avoid electrical breakdown over the edges. The structure of the sensors is presented schematically in Figure $2 b, c$. The CNF sensor was then sandwiched between two electrodes and fixed together from outside of the electrode perimeter using a sticker film (two-way glue, manufactured by Kuretake Co.). A photograph of a fabricated sensor is presented in Figure 2d. The total thickness of the assembled sensor was about $300 \mu \mathrm{m}$, as measured with a micrometer gauge.

In total, four CNF sensor elements with an electrode diameter of $15 \mathrm{~mm}$ were assembled and characterized. In addition, one larger CNF sensor (with diameter of $20 \mathrm{~mm}$ ) was assembled and tested to study the sensitivity distribution over the sensor surface. A polyvinylidene fluoride (PVDF) sensor was assembled using the same method as for the CNF sensors and used as a reference. PVDF has been widely used in mechanical (e.g. pressure, acceleration, vibration and tactile sensors etc.), acoustics and IR sensors ${ }^{24}$, in the application areas including energy 
conversion $^{25}$ and medical measurements ${ }^{26-28}$. Specifically, an un-metallized, $28 \mu \mathrm{m}$ thick PVDF film manufactured by Measurement Specialties Inc. (Hampton, USA) was used in this study ${ }^{29}$.

\subsection{Piezoelectric sensitivity measurements}

A Brüel \& Kjaer Mini-Shaker Type 4810 generating a dynamic excitation force was used in the sensor sensitivity measurements. A sinusoidal input for the shaker was provided with a Tektronix AFG3101 function generator. A commercial high sensitivity dynamic force sensor (PCB Piezotronics, model 209C02) was used as a reference sensor for the dynamic excitation force. A load cell (Measurement Specialties Inc., model ELFS-T3E-20L) was used as reference sensor to measure the static force between the sample and shaker's piston (diameter $4 \mathrm{~mm}$ ). A pre-compression, which produces the static force, is needed to keep the sample in place and to prevent the piston from jumping off the surface during the measurement. Figure 3a shows the setup used to measure the sensor sensitivity. The same measurement principle has been previously used to evaluate the sensor sensitivity of piezoelectric polymer film (PVDF) $26-28,30-33$.

The sensor sensitivity is closely related to the longitudinal piezoelectric coefficient $d_{33}$. The longitudinal $d_{33}$ coefficient describes the electric polarization generated in the same direction as the stress applied ${ }^{34}$. Thus, in the measurement of the sensor sensitivity, the electrodes were located on the top and the bottom of the tested film, which was excited with a dynamic force in the $\mathrm{Z}$ or thickness direction. The sensitivity is defined here as the charge generated by the sensor divided by the normal force used to excite the sensor ( $\mathrm{C} / \mathrm{N}$ units). 
To measure the sensor sensitivity of the four CNF sensors (15 $\mathrm{mm}$ diameter) in the thickness direction, the sensor was placed horizontally on the metal plate, see Figure 2e. The charge developed by the sensor was measured with a custom-made combination of a charge amplifier and a 16-bit $\mathrm{AD}$-converter. The connection to the $\mathrm{AD}$-converter from the sensor was provided via coaxial wires and crimp connectors (Nicomatic Crimpflex). The AD-converter also had additional channels for sampling the voltage signals from the reference sensors. The measured data was processed to solve the sensitivity of the sensor to the force. Since the excitation force was sinusoidal, the sensitivity was calculated simply by dividing the amplitudes of the respective signals. Possible baseline drift in the signals was removed with high-pass filtering before the sinusoidal amplitudes were solved by fitting sinusoidal waveforms to the signals, as described in the IEEE Standard for Digitizing Waveform Recorders (IEEE Std 1241).

A static force of approximately $3 \mathrm{~N}$ was applied. The sensor was excited with dynamic sinusoidal $2 \mathrm{~Hz}$ force input signal of $1.4 \mathrm{~N}$ (peak-to-peak). The excitation was applied in the middle of the sensor. The measurement was repeated three times; between the measurements the static force was relieved and the sensor was re-positioned on the metal plate for a new measurement. The same measurements were conducted from both sides of the sensor, resulting in a total of six excitation rounds per sensor.

\subsection{Measurement of sensor characteristics}

In addition to the piezoelectric sensitivity measurements described in the previous Section, more comprehensive measurements were performed on the CNF sensor with the electrode 
diameter of $20 \mathrm{~mm}$. Likewise, similar measurements were carried out with the reference PVDF sensor. First, the sensitivity distribution over the sensor surface was measured. The CNF and PVDF sensors were excited by applying force to nine different locations on the sensor area, one at a time (see Figure 3b). The same positions were excited from both sides of the sensor, resulting in a total of 18 excitation rounds per sensor.

Next, the charge developed by the CNF and PVDF sensors was measured as a function of dynamic excitation force to determine the nonlinearity of the sensor. The amplitude of the dynamic excitation force was altered from approximately $0.1 \mathrm{~N}$ to $5 \mathrm{~N}$ (peak-to-peak) with a frequency of $2 \mathrm{~Hz}$. Finally, the hysteresis error for the sensors was determined. The sensor hysteresis error is defined as the deviation of the sensor's output at a specified point of the input signal when this point is approached from opposite directions ${ }^{35}$. Here the sensor hysteresis curve was determined by measuring the generated charge as a function of dynamic excitation force, first by increasing the force between the excitation rounds (duration about 10 seconds) and then by decreasing it. At each excitation round, no fatigue in sensor signals was observed during the excitation. The excitation force range was approximately 0.7-2.8 $\mathrm{N}$ (peak-to-peak) with a frequency of $2 \mathrm{~Hz}$.

\section{Results}

\subsection{CNF film characterization}


Cross-sectional and plane view micrographs (SEM) of a CNF film are shown in Figure 4. The cross-section of the film (Figure 4a) reveals a layered, porous structure with an average thickness of $45 \pm 3 \mu \mathrm{m}$. The film structure is similar to those reported in a previous study ${ }^{18}$. The surface view of the film (Figure $4 b$ ) shows a random orientation of the nanofibrils. No traces of defects were found. The density of the CNF film was estimated to be $1.38 \mathrm{~g} / \mathrm{cm}^{3}$, based on measurement of film dimensions and weight measured in ambient air.

The photometric stereo images and the reconstructed surface topography map reveal a prominent streaky structure on the surface of the CNF film. Figure 5a illustrates the surface topography map, showing the typical cloudy and random appearance of webs formed from fiber suspensions. The close-up view (Figure 5b,c) clearly shows a streaky pattern, likely produced by the filter mesh on top of which the CNF dispersion was dewatered to form a film. The distance between the diagonal streaks on the surface is approximately $140 \mu \mathrm{m}$, and the finer regular structure seen on each streak corresponds to the distances between the wires of the dewatering mesh, approximately $48 \mu \mathrm{m}$. The streaky pattern is clearly visible on both sides of the CNF film. 2D spectrum analysis of the photometric stereo based surface topography from an area of several square millimeters confirm the observation made by SEM, which indicated a film with the fibrils randomly oriented in the plane.

The calculated relative permittivity and dielectric loss tangent values for the CNF film obtained from the LCR evaluation are presented as a function of frequency in Figure 6. The deviation span indicate accuracy of the LCR meter for each data point at $100 \mathrm{~Hz}$ to $1 \mathrm{MHz}$. At a $1 \mathrm{kHz}$ frequency the obtained relative permittivity of the CNF film was about 3.47 and the 
dielectric loss $(\tan \delta$ ) about 0.011 . The capacitance of the CNF film varied from $78 \mathrm{pF}$ to $73 \mathrm{pF}$ at $100 \mathrm{~Hz}$ to $1 \mathrm{MHz}$, respectively. The average relative permittivity and dielectric losses were 3.38 and 0.071, respectively, measured with SPDR with nominal frequency of $9.97 \mathrm{GHz}$.

The results for ferroelectric hysteresis are shown in Figure 7. The capacitance or linear component in the polarization data is dominant in the measurements at electric fields from 5 to $15 \mathrm{~V} / \mu \mathrm{m}$, thus showing that the CNF film has no significant ferroelectric hysteresis at low or moderate electric fields (Figure 7a). However, a nonlinear behavior in polarization is detected at electric fields between 40 to $50 \mathrm{~V} / \mu \mathrm{m}$ (Figure $7 \mathrm{~b}$ ). This suggests that the film has some level of ferroelectric properties at high electric fields. The remanent polarization at $50 \mathrm{~V} / \mu \mathrm{m}$ field is small $\left(0.15 \mu \mathrm{C} / \mathrm{cm}^{2}\right)$.

\subsection{Piezoelectric sensitivity of CNF films}

The operation of four nominally identical CNF sensors with diameter of $15 \mathrm{~mm}$ was evaluated with sensitivity measurements (Table 1). The values are presented as mean sensitivity \pm standard deviations for each sensor side. The average sensitivity value for the four CNF sensors was (5.7 $\pm 1.2) \mathrm{pC} / \mathrm{N}$.

Thorough sensor characterization was performed for the larger CNF sensor and the PVDF reference sensor. Figure $3 \mathrm{~b}$ shows the excitation positions for the areal sensitivity distribution measurement. Both sensors had $20 \mathrm{~mm}$ diameter electrodes while the shaker's piston was $4 \mathrm{~mm}$ in diameter, as illustrated in Figure $3 \mathrm{~b}$. Figure 8 shows the CNF and PVDF sensitivity as a 
function of the position of the excitation. Negligible variations in sensitivity over the surface were observed. The average sensitivity for the CNF sensor was found to be $4.7 \pm 0.9 \mathrm{pC} / \mathrm{N}$, while that of the reference PVDF sensor was $27.5 \pm 2.6 \mathrm{pC} / \mathrm{N}$.

The nonlinearity is defined as the maximum deviation of a real transfer function from a linear correlation $^{35}$. Here the nonlinearity for CNF and PVDF sensors was determined by fitting a first degree polynomial via least-squares minimization (Matlab function polyfit). Figure 9a shows the charge generated by the CNF and PVDF sensors as a function of the dynamic excitation force. The respective fitted polynomials for the sensors are shown as dashed (PVDF 34.01x-9.66) and solid (CNF 6.51x-1.23) lines. Instead of the maximum deviation from the linear correlation, the nonlinearity is presented here as the mean \pm standard deviation of data point deviations from the fitted polynomial. The nonlinearity was found to be $(0.86 \pm 0.48) \mathrm{pC}$ and $(6.47 \pm 3.76) \mathrm{pC}$ and for CNF and PVDF sensors, respectively.

Figure $9 \mathrm{~b}$ shows the sensor hysteresis measurements carried out by increasing (solid line and circles) and decreasing (dashed line and crosses) the dynamic excitation force. The maximum difference between the increasing and decreasing measurements was about the same, 0.92 and $0.93 \mathrm{pC}$ for the CNF and PVDF sensors, respectively.

\section{Discussion}


The SEM images revealed a layered, porous structure, resembling that of an electret material, ElectroMechanical Film (EMFi), widely used in sensor applications ${ }^{36,37}$. The surface topography of the CNF film is dominated by streaks, approximately $140 \mu \mathrm{m}$ apart from each other, apparently reproduced from the dewatering fabric mesh ${ }^{18}$. Apart from the mesh-induced pattern, the surface of the film is fairly smooth and the fibrous structure is randomly oriented. The density of the CNF film was found to be $1.38 \mathrm{~g} / \mathrm{cm}^{3}$ which is similar to other reports ${ }^{18}$. In comparison, the densities of the $\mathrm{PVDF}^{29}$ and $\mathrm{EMFi}^{36}$ sensor materials are $1.78 \mathrm{~g} / \mathrm{cm}^{3}$ and 0.33 $\mathrm{g} / \mathrm{cm}^{3}$, respectively. Since the CNF film has a porous structure, it is reasonable that CNF film density is slightly lower that of the PVDF film. On the other hand, the EMFi film has much lower density due to the gas-filled voids separated by polypropylene layers, formed during the manufacturing process.

Relative permittivity of the CNF film was obtained from both LCR and resonator measurements and they match very well, giving a value of about 3.4. Since the measurements were done in ambient conditions, the relative humidity may have caused a small increase in both relative permittivity and dielectric loss, regardless the heat treatment of the films prior to the measurements. However, it is not considered as a significant source of error. The obtained relative permittivity is close to values of dielectric polymers such as polyimide and polytetrafluoroethylene or other organic materials known for their low permittivity ${ }^{38}$. The change in relative permittivity as a function of frequency was clearly seen from the LCR measurements. At the same time, dielectric losses increased rapidly from $10 \mathrm{kHz}$ onward accompanied with steeper declining of relative permittivity, which is typical indication of a relaxation mechanism taking place (Figure 6). However, the change of relative permittivity was quite small, around $7 \%$ 
from $100 \mathrm{~Hz}$ to $1 \mathrm{MHz}$, and it was at similar level as that obtained at $\sim 10 \mathrm{GHz}$, suggesting that only minor level of dielectric relaxation phenomena is occurring due to Maxwell-Wagner-Sillars (MWS) type of the polarization ${ }^{39}$. Such polarization takes place typically at acoustic frequencies and is associated with e.g. immobilized free charges on the interfaces of different media having contrast in conductivity and permittivity. In the case of EMFi, charges are designedly introduced into the gas-filled voids separated by polypropylene layers while in this case they can be byproduct of the manufacturing process, yet having effectively similar function as in EMFi. Since the sensitivity measurements were carried out at low frequencies, the possible contribution of MWS polarization cannot be excluded at this point. Therefore, the actual mechanism behind the observed behavior remains unclear. However, in can be concluded that utilizing high frequency, the dielectric relaxation expands over a wide frequency spectrum compared to that from MWS relaxation.

In addition, it should be pointed out that the used external electrodes pressed against the surfaces of the sample may cause errors in dielectric and ferroelectric measurements. Any air gaps between the electrodes and the sample film may decrease the coupling of the electric field and measured capacitance. The surface contact makes it difficult to distinguish losses from the materials and the contacts. By using two different dielectric measurements, with and without electrodes, reasonable conclusions could be drawn to address these issues.

The ferroelectric hysteresis measurements showed that the CNF film can withstand quite high electric fields, despite its porous microstructure. Small remanence polarization could be found with electric field above $\sim 40 \mathrm{~V} / \mu \mathrm{m}$ and there is an indication that nanocellulose acts like an 
incipient organic ferroelectric material under a very high electric field ${ }^{40}$. The high coercive field compared to similar inorganic materials is a commonly known property of organic ferroelectric materials ${ }^{40}$. It should be noted that the above mentioned issue with electrode contacts may influence also to the ferroelectric results. At high electric fields the electrostriction may also contribute to the polarization while ferroelectricity is expected to be dominant effect, which is discussed more later on. However, these results showed that the investigated CNF films need high electric field for poling and exhibit characteristics of ferroelectricity above $40 \mathrm{~V} / \mu \mathrm{m}$. In such case the observed ferroelectric effect in CNF film results from the permanent dipole moment of $\mathrm{CNCs}$ with some level of orientation resulting from the film fabrication process, which is however not apparent from image analyses. Related observations are found in the recent work by Frka-Petesic et al. who reported experimental evidence of giant permanent electricdipole moment in $\mathrm{CNCs}^{14}$.

The piezoelectric sensors were fabricated from the $45 \mu \mathrm{m}$-thick CNF film and copper electrodes evaporated on a PET substrate. The sensitivity of the fabricated sensors was measured using a dedicated measurement setup. All five (four with electrode diameter of $15 \mathrm{~mm}$ and one of $20 \mathrm{~mm}$ ) prepared piezoelectric CNF sensors showed similar sensitivity values, with sensitivity averages from 4.7 to $6.4 \mathrm{pC} / \mathrm{N}$. These results suggest that the nanofibrils are homogeneously distributed inside the film and the sensitivity values are reliable. Both the CNF and the PVDF reference sensors were found to be rather linear within the used measurement range. Linearly proportional force-charge behavior and spontaneous polarization under applied stress, shown in Figure 9, are both characteristic features of piezoelectric materials. Moreover, although the sensor hysteresis in the CNF sensor was small, it was slightly larger than that of the PVDF 
sensor which also supports the above mentioned observations. Thus, it can be expected that also hysteresis curve in Figure 7 is ferroelectric in nature.

The sensor structure comprising the CNF films sandwiched between the copper electrodes on PET may affect the measured sensitivity values. The contact between the actual sensing material and the electrode is not as good as in the case of electrodes processed directly on the sensing material. In comparison, less variation in sensitivity values were observed when electrodes were either printed or evaporated directly on PVDF film 32,33 .

In 2000 Fukada concluded, that a shear piezoelectric coefficient $d_{14}$ for wood is as low as -0.1 $\mathrm{pC} / \mathrm{N}^{41}$, which suggest poor potential for application as piezoelectric material. However, the case is different for cellulose systems at the nanoscale. There are recent experimental evidences indicating that the orientation of $\mathrm{CNC}$ crystals has a significant effect on the overall piezoelectric response $^{12}$, and that $\mathrm{CNC}$ has a permanent dipole momentum ${ }^{14}$. It was also suggested that regenerated CNC (cellulose II) has the apparent piezoelectric coefficient on the range 35-60 $\mathrm{pC} / \mathrm{N}^{13}$. One should point out that the CNF film studied in this work was not optimized as a piezoelectric material and was not polarized nor oriented. However, it cannot be ruled out that the CNF film fabrication method, including filtering and hot-pressing, may have caused some alignment of the cellulose nanofibrils inside the thick film. The sensor sensitivity obtained for the CNF film in this work is expected to significantly increase by direct assembly of the material for optimal alignment of $\mathrm{CNC}$ dipoles. The poling procedure, in general, consists of applying an electric field on a processed film for a certain period of time in order to generate piezoelectric properties. The poling (or other type orientation) of the CNF film should lead to the polarization 
of the crystalline $\mathrm{CNC}$ regions inside the $\mathrm{CNF}$ film, further leading to a remarkable increase of piezoelectric effect due to the large piezoelectric coefficient of the crystalline components ${ }^{12,14}$. In comparison, a sensitivity of about $27.5 \mathrm{pC} / \mathrm{N}$ was obtained here for a sensor made from a polarized 28- $\mu$ m-thick PVDF film ${ }^{29}$. This is about four times larger than the sensitivity of the CNF film measured in this work. The initial results of orientation of water-processed CNF-films, using DC voltage, suggest the potential of remarkably higher sensitivity values ${ }^{16}$. However, polarization of dry film is difficult due to the entangled and stiff structure of the film and thus, further fabrication process development is required in order to increase the CNF film sensitivity.

\section{Conclusion}

To conclude, self-standing native cellulose nanofibril (CNF) films were synthesized and their microstructure, and dielectric and piezoelectric properties were studied. Image-based analysis, scanning electron microscopy (SEM), and ferroelectric and electromechanical characterizations were carried out for the CNF films. The obtained results suggest that nanocellulose is a promising new piezoelectric material for sensors, actuators and energy generators, which has obvious applications in fields such as sensors, electronics and biomedical diagnostics. 


\section{FIGURES}

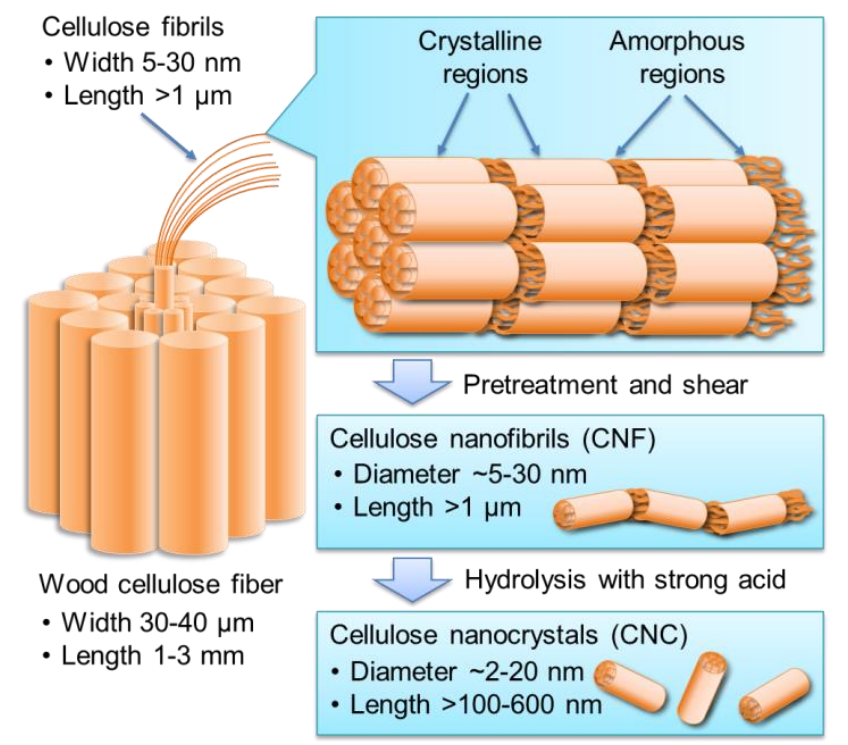

Figure 1. Schematic representation of nanocelluloses obtained from wood cellulose fibers. The cellulose fibers are first deconstructed into microfibrils and after various processing steps into cellulose nanofibrils (CNF). Cellulose nanocrystals (CNC) can be further obtained by using acid hydrolysis. Nanocellulose processing and structure are described in details in the literature ${ }^{1}$. 


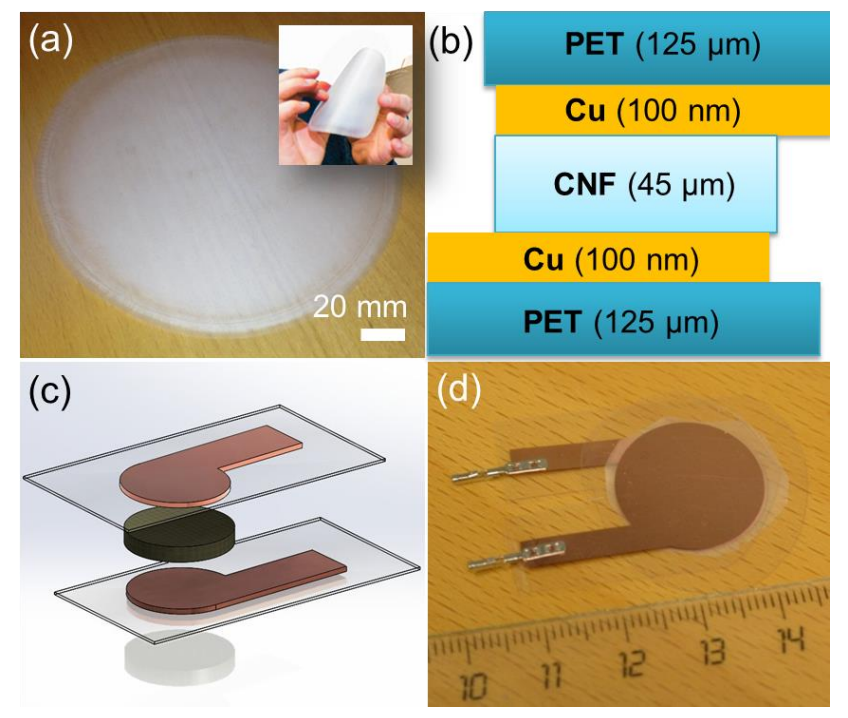

Figure 2. (a) Photographs of a fabricated self-standing CNF film and its bending robustness (an insert). (b,c) A schematic side-view and (d) a photograph of assembled sensor. 


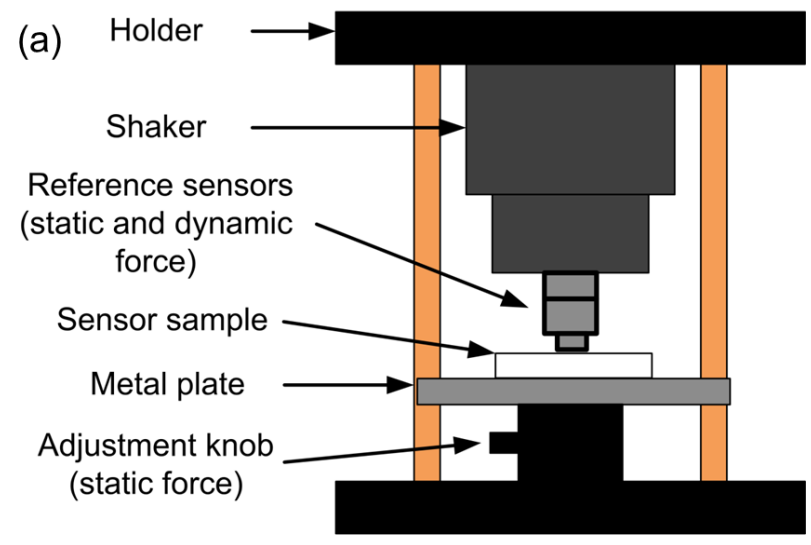

(b)

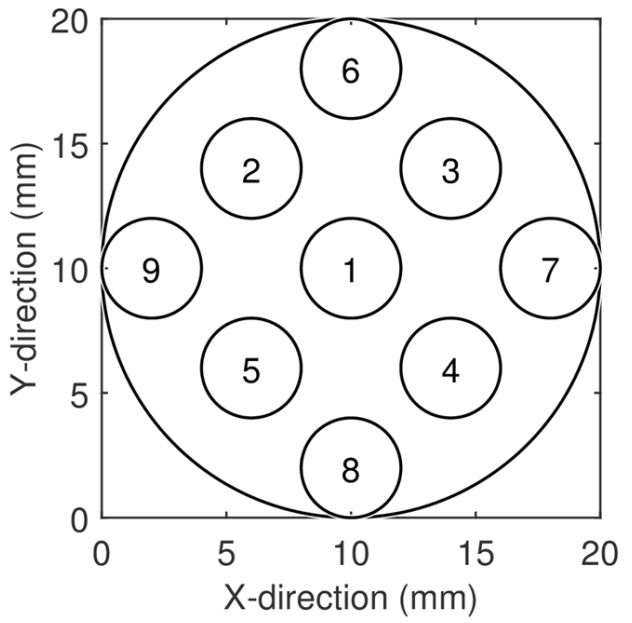

Figure 3. (a) The setup used for sensor sensitivity measurement. (b) The excitation positions for the sensitivity distribution measurements. 

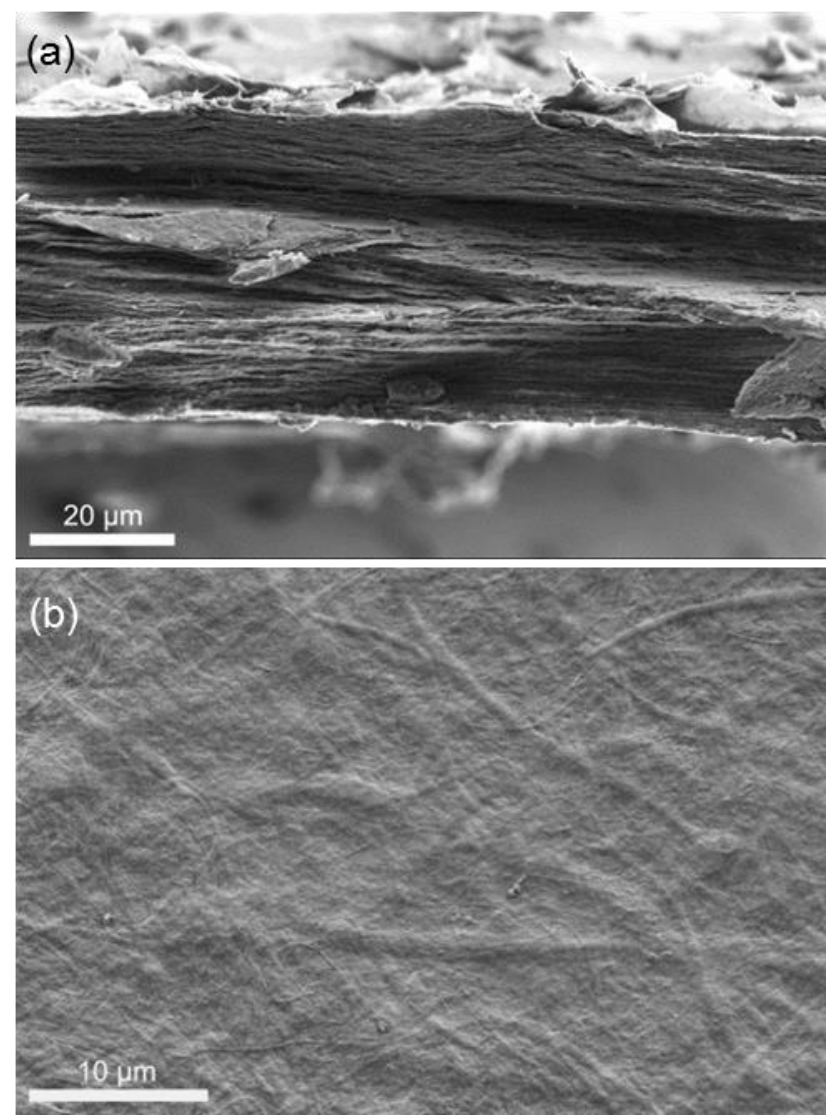

Figure 4. (a) Cross-section and (b) plane view images (SEM) of a CNF film. 

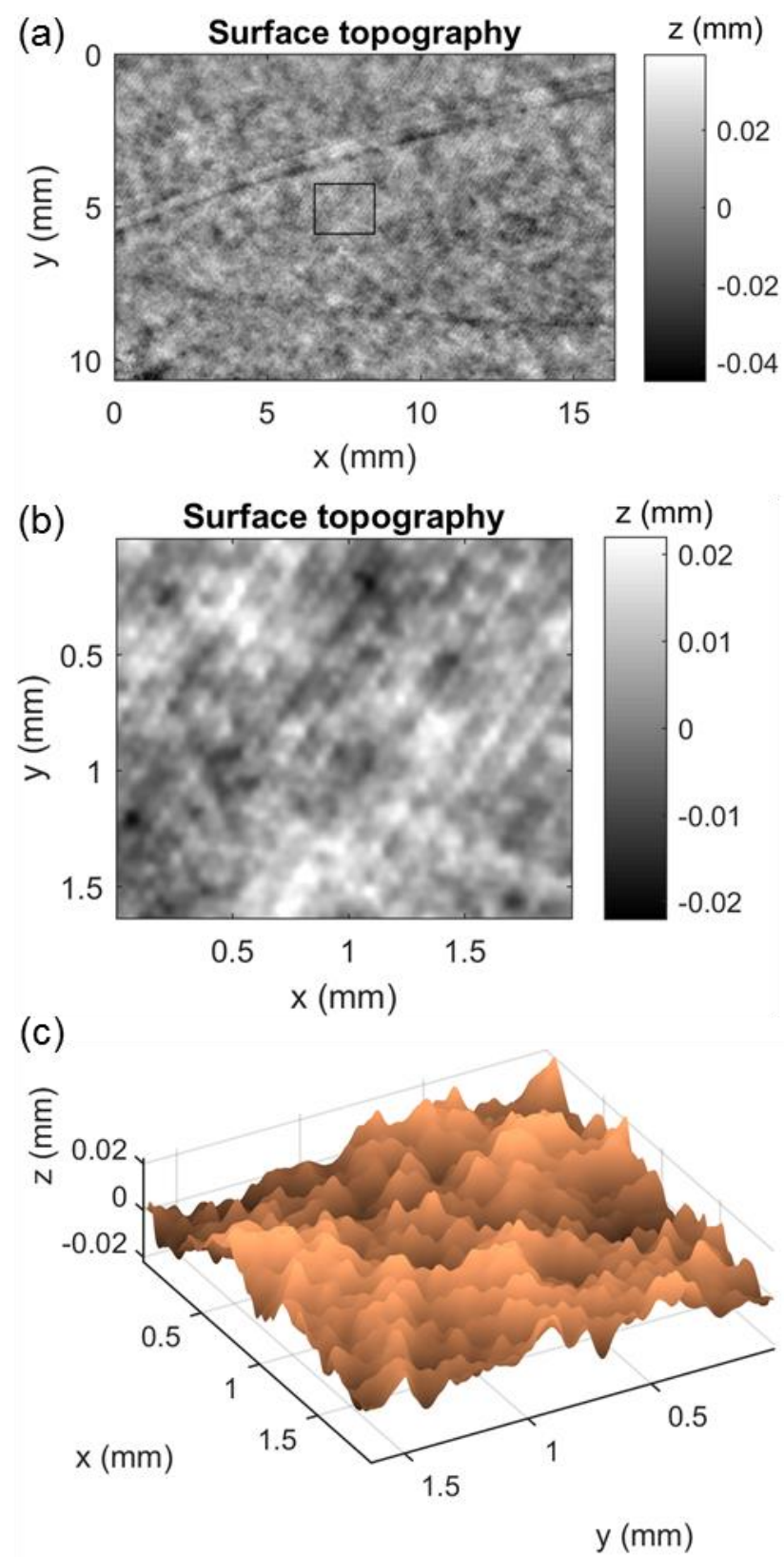

Figure 5. (a) CNF surface topography map and (b) 2D and (c) 3D close-ups (inside the black box in (a)). 


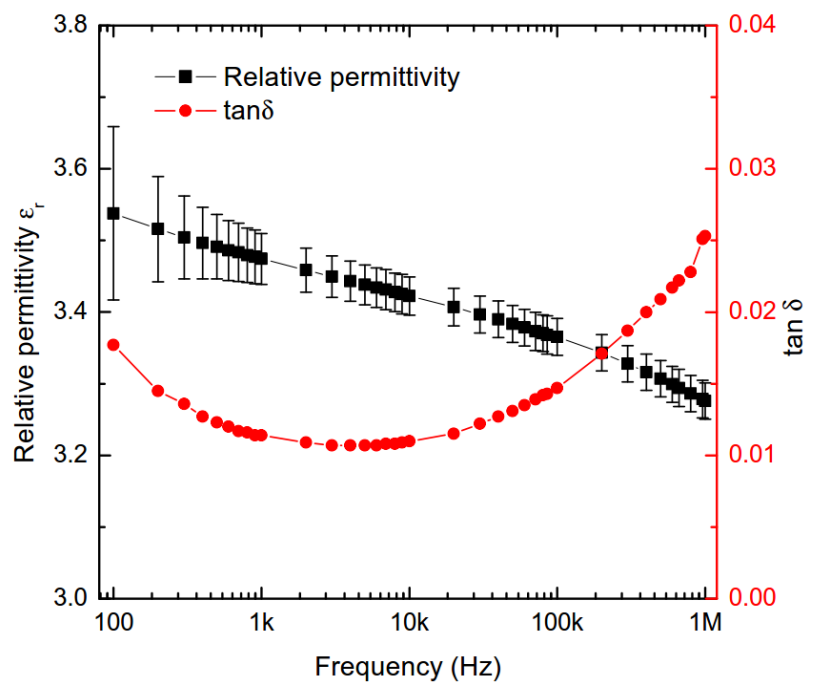

Figure 6. Relative permittivity and loss tangent (tan $\delta$ ) of CNF film obtained from LCR measurements. 

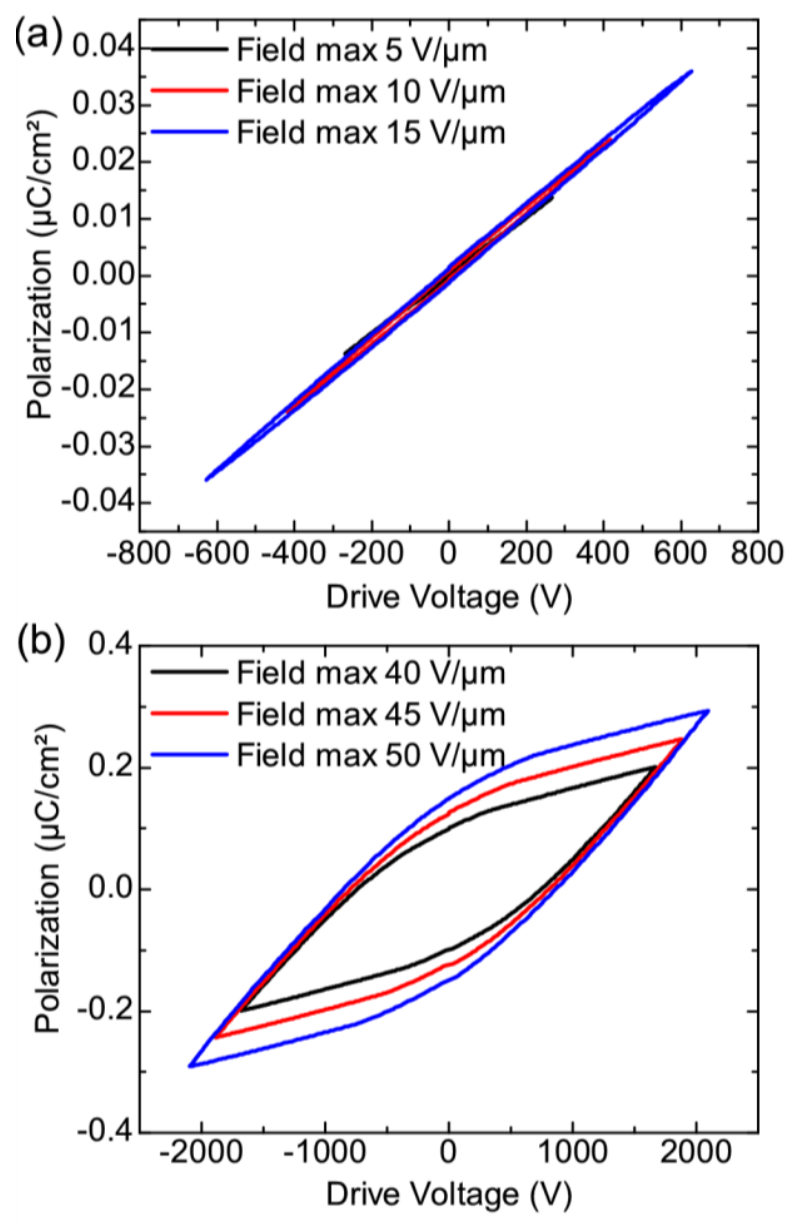

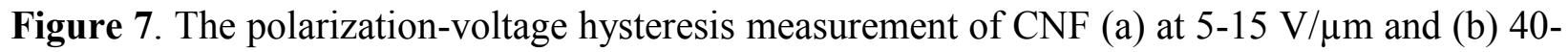
$50 \mathrm{~V} / \mu \mathrm{m}$ electric fields at room temperature. 


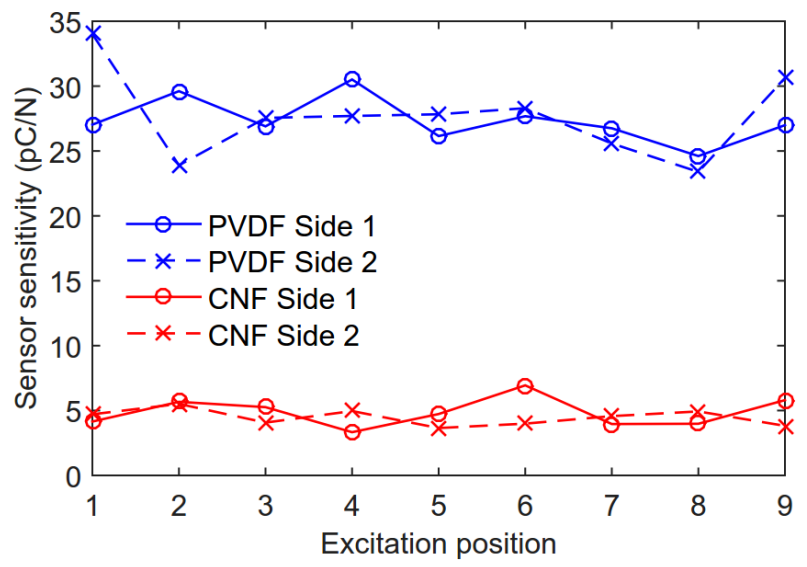

Figure 8. The piezoelectric sensitivity as a function of excitation position. 

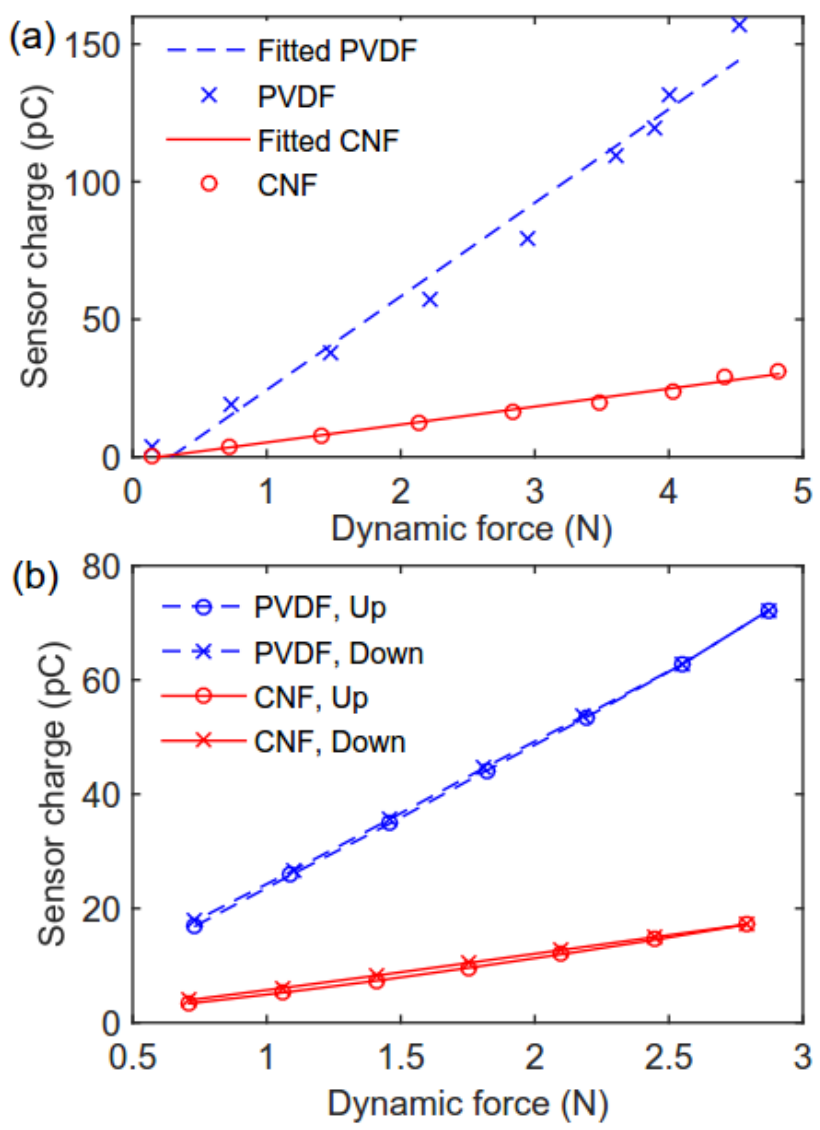

Figure 9. (a) The nonlinearity and (b) hysteresis curves for the CNF and the PVDF reference sensors. 
TABLES.

Table 1. Average force sensitivities for each sensor side.

\begin{tabular}{ccccc}
\hline $\begin{array}{c}\text { Sensor } \\
\text { name }\end{array}$ & $\begin{array}{c}\text { Electrode } \\
\text { diameter }(\mathbf{m m})\end{array}$ & $\begin{array}{c}\text { Sensitivity }(\boldsymbol{p C} / \mathbf{N}), \\
\text { side 1 }\end{array}$ & $\begin{array}{c}\text { Sensitivity }(\boldsymbol{p C} / \mathbf{N}), \\
\text { side 2 }\end{array}$ & $\begin{array}{c}\text { Sensitivity }(\boldsymbol{p C} / \mathbf{N}), \\
\text { average }\end{array}$ \\
\hline $\boldsymbol{S 1}$ & 15 & $4.7 \pm 1.1$ & $5.8 \pm 1.6$ & $5.3 \pm 1.4$ \\
$\boldsymbol{S 2}$ & 15 & $5.9 \pm 0.9$ & $4.5 \pm 0.5$ & $5.2 \pm 1.0$ \\
$\boldsymbol{S 3}$ & 15 & $4.6 \pm 0.4$ & $7.4 \pm 1.6$ & $6.0 \pm 1.9$ \\
$\boldsymbol{S 4}$ & 15 & $6.1 \pm 1.3$ & $6.7 \pm 1.2$ & $6.5 \pm 1.3$ \\
$\boldsymbol{S 5}$ & 20 & $4.9 \pm 1.1$ & $4.5 \pm 0.6$ & $4.7 \pm 0.9$ \\
\hline
\end{tabular}




\section{AUTHOR INFORMATION}

\section{Corresponding Author}

*Phone: +358 40541 5276, Email: sampo.tuukkanen@tut.fi.

\section{Author Contributions}

MV has prepared the CNF films. MM has performed the image based analysis for the CNF films. ES has performed the scanning electron microscopy analysis. AP and ST have fabricated the piezoelectric sensors. SR has performed the piezoelectric sensitivity measurements and analyzed the results with ST. TS have performed the dielectric properties and ferroelectric hysteresis measurements and analyzed the results with JJ. SR and ST wrote the most of the manuscript. All authors commented the manuscript and gave their approval to the final version of the manuscript. $\$$ These authors contributed equally.

\section{Notes}

The authors declare no competing financial interest.

\section{ACKNOWLEDGMENT}

The authors acknowledge funding from the Academy of Finland (Dec. No. 137669, 258124, 264743, 267573 and 273663). Author TS appreciatively acknowledge the Riitta ja Jorma J. Takanen, KAUTE, Tauno Tönning and Ulla Tuominen foundations as well as Infotech Oulu Doctoral Program for financial support. 


\section{REFERENCES}

(1) Moon, R. J.; Martini, A.; Nairn, J.; Simonsen, J.; Youngblood, J. Cellulose Nanomaterials Review: Structure, Properties and Nanocomposites. Chem. Soc. Rev. 2011, 40 (7), 3941.

(2) Vuorinen, T.; Zakrzewski, M.; Rajala, S.; Lupo, D.; Vanhala, J.; Palovuori, K.; Tuukkanen, S. Printable, Transparent, and Flexible Touch Panels Working in Sunlight and Moist Environments. Adv. Funct. Mater. 2014, 24 (40), 6340-6347.

(3) Lehtimäki, S.; Tuukkanen, S.; Pörhönen, J.; Moilanen, P.; Virtanen, J.; Honkanen, M.; Lupo, D. Low-Cost, Solution Processable Carbon Nanotube Supercapacitors and Their Characterization. Appl. Phys. A 2014, 117 (3), 1329-1334.

(4) Rim, Y. S.; Bae, S.-H.; Chen, H.; De Marco, N.; Yang, Y. Recent Progress in Materials and Devices toward Printable and Flexible Sensors. Adv. Mater. 2016.

(5) Isoniemi, T.; Tuukkanen, S.; Cameron, D. C.; Simonen, J.; Toppari, J. J. Measuring Optical Anisotropy in poly(3,4-Ethylene Dioxythiophene):poly(styrene Sulfonate) Films with Added Graphene. Org. Electron. 2015, 25, 317-323.

(6) Tuukkanen, S.; Lehtimaki, S.; Jahangir, F.; Eskelinen, A.-P.; Lupo, D.; Franssila, S. Printable and Disposable Supercapacitor from Nanocellulose and Carbon Nanotubes. In Proceedings of the 5th Electronics System-integration Technology Conference (ESTC); IEEE, 2014; pp 1-6.

(7) Torvinen, K.; Lehtimäki, S.; Keränen, J. T.; Sievänen, J.; Vartiainen, J.; Hellén, E.; Lupo, D.; Tuukkanen, S. Pigment-Cellulose Nanofibril Composite and Its Application as a Separator-Substrate in Printed Supercapacitors. Electron. Mater. Lett. 2015, 11 (6), 1040- 
1047.

(8) Lehtimäki, S.; Suominen, M.; Damlin, P.; Tuukkanen, S.; Kvarnström, C.; Lupo, D. Preparation of Supercapacitors on Flexible Substrates with Electrodeposited PEDOT/Graphene Composites. ACS Appl. Mater. Interfaces 2015, 7 (40), 22137-22147.

(9) Harrison, J. S.; Ounaies, Z. Piezoelectric Polymers. In Encyclopedia of Polymer Science and Technology; John Wiley \& Sons, Inc.: Hoboken, NJ, USA, 2002; Vol. 3.

(10) Fukada, E. Piezoelectricity of Wood. J. Phys. Soc. Japan 1955, 10 (2), 149-154.

(11) Fukada, E. Piezoelectricity as a Fundamental Property of Wood. Wood Sci. Technol. 1968, $2(4), 299-307$.

(12) Csoka, L.; Hoeger, I. C.; Rojas, O. J.; Peszlen, I.; Pawlak, J. J.; Peralta, P. N. Piezoelectric Effect of Cellulose Nanocrystals Thin Films. ACS Macro Lett. 2012, 1 (7), 867-870.

(13) Cheng, H. FlexoElectric Nanobiopolymers (FEPs) Exhibiting Higher Mechanical Strength (7.5 GPa), Modulus (250 GPa), and Energy Transfer Efficiency (75\%). Worldw. Electroact. Polym. (Artificial Muscles) Newsl. 2008, 10 (2), 5-7.

(14) Frka-Petesic, B.; Jean, B.; Heux, L. First Experimental Evidence of a Giant Permanent Electric-Dipole Moment in Cellulose Nanocrystals. EPL (Europhysics Lett. 2014, 107 (2), 28006.

(15) Rajala, S.; Vuoriluoto, M.; Rojas, O. J.; Franssila, S.; Tuukkanen, S. Piezoelectric Sensitivity Measurements of Cellulose Nanofibril Sensors. In Proc. XXI IMEKO World Congr.; Prague, Czech Republic, 2015; pp 2-6. 
(16) Tuukkanen, S.; Rajala, S. A Survey of Printable Piezoelectric Sensors. In Proc. IEEE SENSORS Conference; IEEE: Busan, South Korea, 2015; pp 1-4.

(17) Pääkko, M.; Ankerfors, M.; Kosonen, H.; Nykänen, a.; Ahola, S.; Österberg, M.; Ruokolainen, J.; Laine, J.; Larsson, P. T.; Ikkala, O.; Lindström, T. Enzymatic Hydrolysis Combined with Mechanical Shearing and High-Pressure Homogenization for Nanoscale Cellulose Fibrils and Strong Gels. Biomacromolecules 2007, 8 (6), 1934-1941.

(18) Österberg, M.; Vartiainen, J.; Lucenius, J.; Hippi, U.; Seppälä, J.; Serimaa, R.; Laine, J. A Fast Method to Produce Strong NFC Films as a Platform for Barrier and Functional Materials. ACS Appl. Mater. Interfaces 2013, 5 (11), 4640-4647.

(19) Woodham, R. J. Photometric Method for Detemining Surface Orientation from Multiple Images. Opt. Eng. 1980, 19 (1), 139-144.

(20) Goldman, D. B.; Curless, B.; Hertzmann, A.; Seitz, S. M. Shape and Spatially-Varying BRDFs from Photometric Stereo. IEEE Trans. Pattern Anal. Mach. Intell. 2010, 32 (6), $1060-1071$.

(21) Mettänen, M. Measurement of Print Quality: Joint Statistical Analysis of Paper Topography and Print Defects, Tampere University of Technology, 2010.

(22) Sohaib, A.; Farooq, A. R.; Atkinson, G. a; Smith, L. N.; Smith, M. L.; Warr, R. In Vivo Measurement of Skin Microrelief Using Photometric Stereo in the Presence of Interreflections. J. Opt. Soc. Am. A. Opt. Image Sci. Vis. 2013, 30 (3), 278-286.

(23) Frankot, R. T.; Chellappa, R. Method for Enforcing Integrability in Shape From Shading Algorithms. IEEE Trans. Pattern Anal. Mach. Intell. 1988, 10 (4), 439-451. 
(24) Harsányi, G. Polymer Films in Sensor Applications: A Review of Present Uses and Future Possibilities. Sens. Rev. 2000, 20 (2), 98-105.

(25) Lang, S. B.; Muensit, S. Review of Some Lesser-Known Applications of Piezoelectric and Pyroelectric Polymers. Appl. Phys. A Mater. Sci. Process. 2006, 85 (2), 125-134.

(26) Rajala, S.; Lekkala, J. Film-Type Sensor Materials PVDF and EMFi in Measurement of Cardiorespiratory Signals a Review. IEEE Sens. J. 2012, 12 (3), 439-446.

(27) Tuukkanen, S.; Julin, T.; Rantanen, V.; Zakrzewski, M.; Moilanen, P.; Lilja, K. E.; Rajala, S. Solution-Processible Electrode Materials for a Heat-Sensitive Piezoelectric Thin-Film Sensor. Synth. Met. 2012, 162 (21-22), 1987-1995.

(28) Kärki, S.; Lekkala, J.; Kuokkanen, H.; Halttunen, J. Development of a Piezoelectric Polymer Film Sensor for Plantar Normal and Shear Stress Measurements. Sensors Actuators, A Phys. 2009, 154 (1), 57-64.

(29) Measurement Specialties Inc. Piezo film sensors, Technical manual http://www.measspec.com (accessed May 5, 2014).

(30) S. Kärki, M. Kiiski, M. Mäntysalo, J. L. A PVDF Sensor with Printed Electrodes for Normal and Shear Stress Measurements on Sole. In Proc. XIX IMEKO World Congr.; Lisbon, Portugal, 2009; pp 1765-1769.

(31) Tuukkanen, S.; Julin, T.; Rantanen, V.; Zakrzewski, M.; Moilanen, P.; Lupo, D. LowTemperature Solution Processable Electrodes for Piezoelectric Sensors Applications. Jpn. J. Appl. Phys. 2013, 52 (5S1), 05DA06.

(32) Rajala, S. N. K.; Mettanen, M.; Tuukkanen, S. Structural and Electrical Characterization 
of Solution-Processed Electrodes for Piezoelectric Polymer Film Sensors. IEEE Sens. J. 2016, $16(6), 1692-1699$.

(33) Rajala, S.; Tuukkanen, S.; Halttunen, J. Characteristics of Piezoelectric Polymer Film Sensors With Solution-Processable Graphene-Based Electrode Materials. IEEE Sens. J. 2015, 15 (6), 3102-3109.

(34) Ramadan, K. S.; Sameoto, D.; Evoy, S. A Review of Piezoelectric Polymers as Functional Materials for Electromechanical Transducers. Smart Mater. Struct. 2014, 23 (3), 033001.

(35) Fraden, J. Handbook of Modern Sensors: Physics, Designs, and Applications, 2nd Ed.; 2010.

(36) Paajanen, M.; Lekkala, J.; Kirjavainen, K. ElectroMechanical Film (EMFi) - a New Multipurpose Electret Material. Sensors Actuators A Phys. 2000, 84 (1-2), 95-102.

(37) Barna, L., Koivuluoma, M., Hasu, M., Tuppurainen, J. and Värri, A. The Use of Electromechanical Film (EMFi) Sensors in Building a Robust Touch-Sensitive Tablet-like Interface. IEEE Sens. J. 2007, 7 (1), 74-80.

(38) DuPont, DuPont Kapton FPC Polyimide Film, Technical Data Sheet http://www.dupont.com/content/dam/dupont/products-and-services/membranes-andfilms/polyimde-films/documents/DEC-Kapton-FPC-datasheet.pdf (accessed Mar 2, 2016).

(39) Perrier, G.; Bergeret, A. Polystyrene-Glass Bead Composites: Maxwell-Wagner-Sillars Relaxations and Percolation. J. Polym. Sci. Part B Polym. Phys. 1997, 35 (9), 1349-1359.

(40) Horiuchi, S.; Tokura, Y. Organic Ferroelectrics. Nat. Mater. 2008, 7 (5), 357-366. 
(41) Fukada, E. History and Recent Progress in Piezoelectric Polymers. IEEE Trans. Ultrason. Ferroelectr. Freq. Control 2000, 47 (6), 1277-1290. 
Table of Contents Graphics

Nanofibrillated cellulose (CNF) film characterization

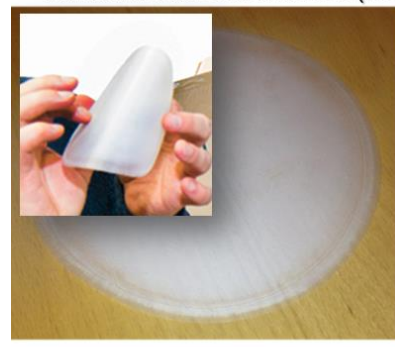

Topography

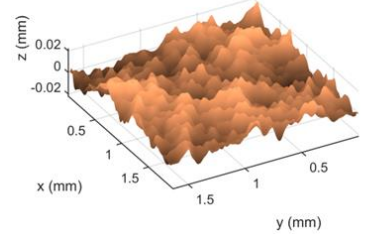

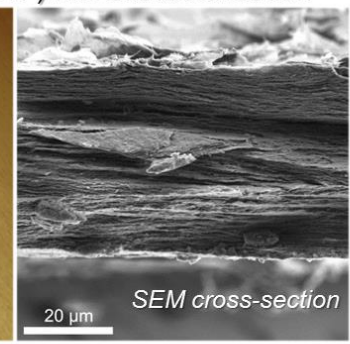

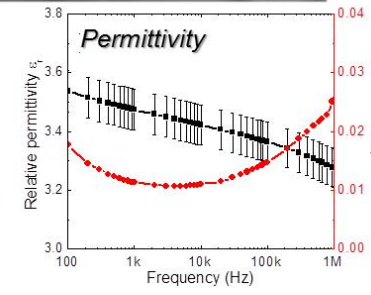

Piezoelectric nanocellulose sensors
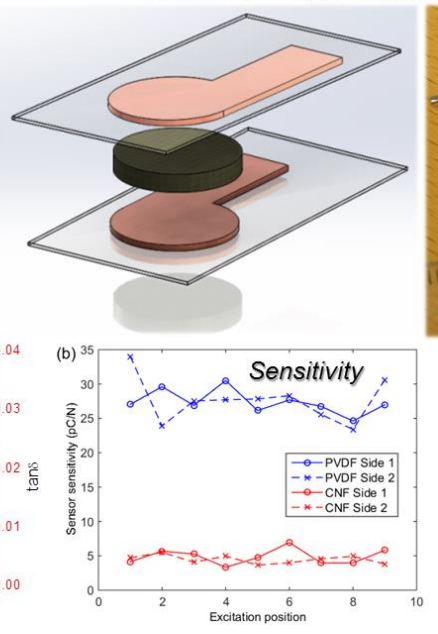
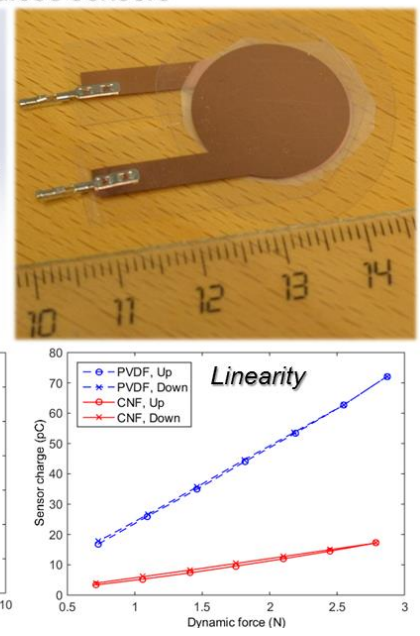